\title{
Evaluación mecánica y mineralógica de un cemento de metacaolín utilizando el método de superficie de respuesta
}

\author{
$\triangle$ Carlos Arturo Díaz-Villamil ${ }^{1}$ \\ Albert Leonard Alzate-Ramirez ${ }^{1}$ \\ Yhan PAUl ARIAS-JARAMILlo ${ }^{1}$
}

\section{Resumen}

En este trabajo, un cemento alcalino con base en metacaolín colombiano (MK) es desarrollado. Los activadores usados: $15 \%$ en masa de $\mathrm{Na}_{2} \mathrm{SiO}_{3}$ y $\mathrm{Na}(\mathrm{OH})$ y $\mathrm{Ca}(\mathrm{OH})_{2}$ se variaron en diferentes concentraciones molares siguiendo un diseño experimental estadístico con modelo de superficie de respuesta. La variable respuesta, resistencia mecánica a compresión, se evaluó en especímenes de pasta cilíndricos a 7 y 14 días de curado a 40 C. Los ensayos de difracción de rayos X identificaron las fases mineralógicas y cementantes presentes en la pasta producto de la síntesis alcalina.

Los resultados analizados mediante software estadísticos permitieron, a través de la ANOVA, identificar un alto nivel de significancia en el factor $\mathrm{Na}(\mathrm{OH})$ así como el efecto creciente en la variable respuesta, mientras que el Ca(OH) ${ }_{2}$ presentó un efecto de curvatura en la región explorada. Las resistencias mecánicas logradas del orden de 34 MPa dan

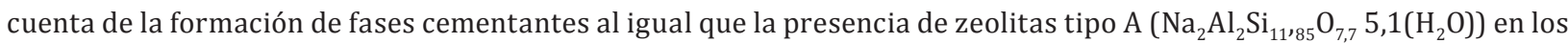
resultados DRX.

Palabras Clave: Activación alcalina, relaciones molares, cementos, metacaolín, DOE.

\section{Mechanical and mineralogical evaluation of a metakaolin cement using the surface response method}

\section{Abstract}

In this work, an alkaline cement based on Colombian metakaolin (MK) is developed. The activators used: $15 \%$ by mass of $\mathrm{Na}_{2} \mathrm{SiO}_{3}$ and $\mathrm{Na}(\mathrm{OH})$ and $\mathrm{Ca}(\mathrm{OH})_{2}$ were varied in different molar concentrations following a statistical experimental design with response surface model. The variable response, mechanical resistance to compression, was evaluated in

$1 \quad$ Universidad de Medellín. Medellín, Colombia.

Autor de correspondencia: Díaz-Villamil, C.A. (Carlos Arturo): Universidad de Medellín, Carrera 87 \#30-65, Medellín, Colombia, +57-4-3405216. Tel +57-320 614 8259. Correo electrónico: cadiaz@udem.edu.co,
Historia del artículo:

Artículo recibido: 10-VI-2019/ Aprobado: 15-I-2020

Disponible online: 15 de enero de 2020

Discusión abierta hasta septiembre de 2021 
cylindrical paste specimens at 7 and 14 days of curing at $40^{\circ} \mathrm{C}$. The X-ray diffraction tests identified the mineralogical and cementing phases present in the pulp product of the alkaline synthesis.

The results analyzed through statistical software allowed, through the ANOVA, to identify a high level of significance in the $\mathrm{Na}(\mathrm{OH})$ factor as well as the increasing effect in the response variable, while the $\mathrm{Ca}(\mathrm{OH})_{2}$ presented a curvature effect in the explored region. Mechanical strengths in the order of $34 \mathrm{MPa}$ account for the formation of cementing phases as well as the presence of type A zeolites $\left(\mathrm{Na}_{2} \mathrm{Al}_{2} \mathrm{Si}_{11.85} \mathrm{O}_{7.7} 5.1\left(\mathrm{H}_{2} \mathrm{O}\right)\right)$ in the result DRX.

Keywords: Alkaline activation, molar ratios, cements, metakaolin, DOE.

\section{Avaliação mecânica e mineralógica de um cimento de metacaulim pelo método de resposta superficial}

\section{Resumo}

Neste trabalho, um cimento alcalino com base em metacaulino colombiano (MK) é desenvuelto. Os ativadores usados: $15 \%$ em massa $\mathrm{Na}_{2} \mathrm{SiO}_{3}$ e $\mathrm{Na}(\mathrm{OH})$ e $\mathrm{Ca}(\mathrm{OH})_{2}$ foram variados em diferentes concentrações molares na sequência de um desenho experimental estatística com modelo de superfície de resposta. A resposta variável, resistência mecânica à compressão, foi avaliada em amostras de pasta cilíndrica aos 7 e 14 dias de cura a $40^{\circ} \mathrm{C}$. Os testes de difração de raios-X identificaram as fases mineralógicas e de cimentação presentes na pasta da síntese alcalina.

Os resultados analisados por meio de software estatístico permitiram, através da ANOVA, identificar um alto nível de significância no fator $\mathrm{Na}(\mathrm{OH})$, bem como o efeito crescente na variável resposta, enquanto o $\mathrm{Ca}(\mathrm{OH})_{2}$ apresentou um efeito de curvatura na região explorada. Forças mecânicas da ordem de 34 MPa são responsáveis pela formação das fases de cimentação bem como pela presença de zeólitas do tipo $\mathrm{A}\left(\mathrm{Na}_{2} \mathrm{Al}_{2} \mathrm{Si}_{11.85} \mathrm{O}_{7.7} 5.1\left(\mathrm{H}_{2} \mathrm{O}\right)\right)$ nos resultados de DRX

Palavras chave: Ativação alcalina, relação molar, cimentos, metacaulim, DOE.

\section{Introducción}

En las últimas cuatro décadas se ha incrementado un interés por investigar nuevos materiales que generen productos adecuados para las construcciones civiles, que sean durables y amigables con el ambiente. (Alonso S. \& Palomo A., 2000) (Yip C.K., Lukey G.C., \& van Deventer J.S.J., 2004) (Bernal S.A., Mejía R., Provis J.L., \& Rose V., 2010).

Algunos investigadores consideran a los Cementos Activados Alcalinamente (CAA) como la tercera generación del cemento; después de la cal y el cemento Pórtland (CP), son una alternativa que parece tener una mayor durabilidad y un mejor desempeño ambiental, teniendo diversas aplicaciones en la industria agrícola, el transporte, la minería, en pozos petroleros y para encapsular desechos radiactivos, entre otros. (Li, Sun, \& Li, 2010). Los CAA están compuestos básicamente de silicio y aluminio (puzolanas) y pueden ser activados con soluciones básicas o también llamadas soluciones alcalinas. (Davidovits, 1991), (Provis, Palomo, \& Shi, 2015).

Los conglomerantes alcalinos inician su historia en 1930 con Khul al investigar el fraguado de las escorias de alto horno en presencia de una disolución de $\mathrm{K}(\mathrm{OH})$, en 1937 Chassevent investiga la reactividad en escorias de alto horno con disoluciones de $\mathrm{K}(\mathrm{OH})$ y $\mathrm{Na}(\mathrm{OH})$. Para 1940 Purdon sintetiza las escorias de alto horno libres de clinker y disoluciones de $\mathrm{NaOH}$ y sales alcalinas. En 1957 Gluwkowsky realiza una síntesis de cementos mediante arcillas (aluminatos hidratados) con rocas vítreas, escorias de alto horno (anhidros) y propone cementos con el sistema $\mathrm{Me}_{2} \mathrm{O}-\mathrm{MeO}-\mathrm{Al}_{2} \mathrm{O}_{3}-\mathrm{SiO}_{2}-\mathrm{H}_{2} \mathrm{O}$ denominado "soil cement". La propuesta de la activación de los cementos alcalinos realizada por Glukhovsky en 1957 es el postulado mejor aceptado por la comunidad científica. Uno de los productos principales de la síntesis 
de cementos alcalinos es el gel NASH quien es el responsable de que la pasta del material cementante junto con el conglomerante adquieran resistencia mecánica a la compresión, éste, es definido como un silicoaluminato o aluminosilicato alcalino (sódico) hidratado. (Palomo, y otros, 2014).

Gluwkowsky propuso que la primera etapa de la formación de un gel NASH se llamara destruccióncoagulación. En donde los iones $(\mathrm{OH})$ provenientes de metales alcalinos, rompen los enlaces covalentes $\mathrm{Si}-\mathrm{O}-\mathrm{Si}$ y Al-O-Al. Dado que los $(\mathrm{OH})^{-}$provocan una redistribución de la densidad electrónica se obtienen grupos silanol ( $\left.\mathrm{Si}^{-}-\mathrm{OH}\right)$ y sialatos $\left(\mathrm{Si}^{-} \mathrm{O}^{-}\right)$. La presencia del catión metálico $\mathrm{Na}^{+}$estabiliza las cargas formando (Si-O)- $\left(\mathrm{Na}^{+}\right)$que impiden que la reacción sea reversible, estos son estables en medios alcalinos y adecuados para el transporte de estructuras reactivas facilitando el desarrollo de la estructura coagulada. En la segunda etapa la llamó coagulación-condensación y se presentan acumulaciones de los productos generados en la primera etapa, provocando el desarrollo de una estructura de cadenas poliméricas por medio de la unión monomérica, denominado proceso de policondensación. La formación del hidroaluminogel está relacionado con el pH del medio. La tercera y última etapa es llamada condensacióncristalización. Las partículas de la fase inicial junto con las micro partículas de la condensación favorecen la precipitación del producto, pero son la composición mineralógica y química de la fase inicial y los componentes del activador los que definen la calidad y cantidad del producto (Criado, 2007). A inicios de 1980 Davidovits usa el mismo sistema, pero con metacaolín en diferentes proporciones de caliza. (Palomo, y otros, 2014).

En el presente trabajo se consideró importante definir las condiciones adecuadas para la activación alcalina, debido a que estos cementos constituyen un sistema complejo de óxidos de aluminio, silicio, sodio y calcio. Por lo anterior se definió usar la metodología de la superficie de respuesta, la cual busca optimizar la variable respuesta; en este caso las mejores resistencias a la compresión simple (RCS), afectada por las variables predictoras como lo son: concentración molar del activador $(\mathrm{NaOH})$ junto con el porcentaje de $\mathrm{Ca}(\mathrm{OH})_{2}$ y de esta forma identificar los cambios en la variable respuesta.

\section{Caracterización de materiales}

En las Tablas 1 y 2 se presentan las características físico químicas del precursor utilizado. La composición mineralógica del MK se identificó haciendo uso de un difractometro de rayos X de polvo, Philips PW 1800 con radiación de cobre $(\mathrm{Cu})(40 \mathrm{kV}, 30 \mathrm{~mA})$ con velocidad de exploración de $2^{\circ}$ por minuto. Figura 1.

\section{TABLA 1. CARACTERÍSTICAS FÍSICAS DEL PRECURSOR}

\begin{tabular}{l|l|l}
\multicolumn{1}{c|}{ Característica física } & \multicolumn{1}{|c|}{ Especificaciones } & \multicolumn{1}{|c}{ Valor } \\
\hline $\mathrm{Dv_{10 } \mu \mathrm { m }}$ & $0,50-1,50 \mu \mathrm{m}$ & $0,70 \mu \mathrm{m}$ \\
\hline $\mathrm{Dv_{50 } \mu \mathrm { m }}$ & $9,50-11,50 \mu \mathrm{m}$ & $10,0 \mu \mathrm{m}$ \\
\hline $\mathrm{Dv_{90 } \mu \mathrm { m }}$ & $36,00-40,00 \mu \mathrm{m}$ & $37,82 \mu \mathrm{m}$ \\
\hline $\begin{array}{l}\text { Retenido sobre malla } \\
\text { U.S. No.325 }\end{array}$ & $<3,0 \%$ & $2,0 \%$ \\
\hline $\begin{array}{l}\text { Área superficial } \\
\text { especifica de Blaine } \\
\text { (NTC-33) }\end{array}$ & $\begin{array}{l}20.000-24.000 \\
\mathrm{~cm}^{2} / \mathrm{g}\end{array}$ & $\begin{array}{l}22.335 \\
\mathrm{~cm}^{2} / \mathrm{g}\end{array}$ \\
\hline Gravedad específica & $2,45-2,65$ & 2,54 \\
\hline Humedad & $1,0-2,0 \%$ & $1,5 \%$ \\
\hline Color & Gris / Gris claro & \\
\hline
\end{tabular}

TABLA 2. PROPIEDADES QUÍMICAS DEL PRECURSOR

\begin{tabular}{l|c|c}
\hline \multicolumn{1}{c|}{ Propiedades químicas } & Rango aceptable & Valor \\
\hline$\% \mathrm{SiO}_{2}$ & $55,0-65,0 \%$ & $54,0 \%$ \\
\hline$\% \mathrm{Al}_{2} \mathrm{O}_{3}$ & $26,5-43,5 \%$ & $40,4 \%$ \\
\hline$\% \mathrm{Fe}_{2} \mathrm{O}_{3}$ & $<5,0 \%$ & $0,90 \%$ \\
\hline$\% \mathrm{SiO}_{2}+\% \mathrm{Al}_{2} \mathrm{O}_{3}+\mathrm{Fe}_{2} \mathrm{O}_{3}$ & $>70 \%$ & $95,95 \%$ \\
\hline $\begin{array}{l}\text { Pérdidas por ignición, } \\
\mathrm{PPI} a 800^{\circ} \mathrm{C}\end{array}$ & $<10 \%$ & $1,4 \%$ \\
\hline$\% \mathrm{SO}_{3}$ & $<4,0$ & $0,10 \%$ \\
\hline
\end{tabular}

La presencia del pico agudo en el DRX del MK en la posición $2 \theta$ de $26,593^{\circ}$ corresponde a la presencia de cristales de cuarzo $(\theta)$, identificado según la base de datos Power Difraction Data (PDF 01-083-0539) (Figura 1). Además, se halló moscovita ( $\mu$ ), código PDF 00-007-0042, que es un filosilicato de la serie isomorfa. La moscovita se considera un mineral no deseado al momento de realizar RCS, ya que puede favorecer planos de falla por la formación laminar y como no se descompone fácilmente, afecta la RCS cuando está presente en los productos de reacción de materiales cementantes. Por 
otra parte, el hombro o halo que sobresale en la región de $2 \theta$ entre los ángulos $15^{\circ}$ y $35^{\circ}$ del DRX, indican que el MK tiene alto grado amorfo ideal para ser activado (Diamond, 1982).

En cuanto a los activadores usados para preparar las soluciones corresponden a; hidróxido de calcio de (93\% de pureza), hidróxido de sodio en lajas grado comercial de (98\% de pureza) y silicato de sodio con $\left(\mathrm{SiO}_{2}=28,1 \%, \mathrm{Na}_{2} \mathrm{O}=8,2 \%, \mathrm{H}_{2} \mathrm{O}=63,7 \%\right)$.

Figura 1. Difractograma de rayos $X$ en el metacaolín sin activar. $\theta$ : cuarzo y $\mu$ : moscovita

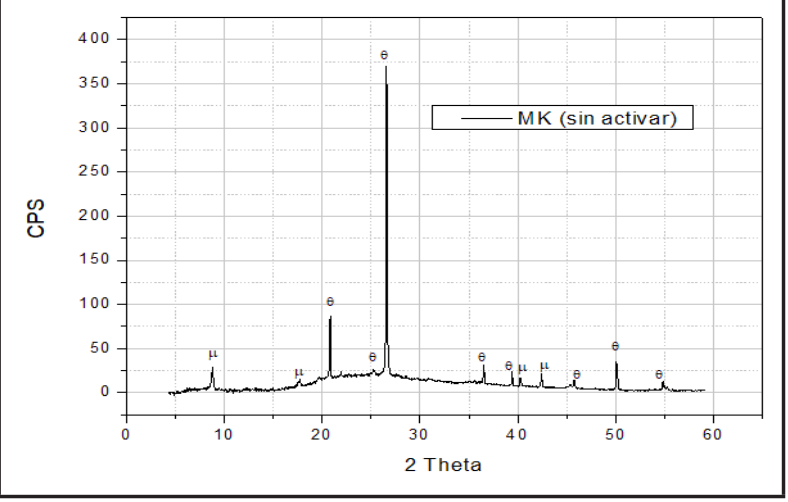

\section{Matriz experimental para elaborar un CAA}

Se plantea un diseño experimental con superficie de respuesta con dos variables predictoras; $\boldsymbol{X}_{1}$ como: concentración molar del $\mathrm{NaOH}$ y $\boldsymbol{X}_{2}$ que representa la masa del $\mathrm{Ca}(\mathrm{OH})_{2}$ con los respectivos niveles, ver Tabla 3. Como variable respuesta se considera la resistencia a la compresión simple y se expresa mediante la Ecuación 1 de regresión lineal, donde $\boldsymbol{Y}$ representa la RCS en $\mathrm{MPa}$; los valores $\beta_{\mathrm{i}}$ son los coeficientes del modelo con valores i $=1,2,3,4,5 ; \boldsymbol{X}_{1}^{2}$ y $\boldsymbol{X}_{2}^{2}$ son los efectos cuadráticos; $\boldsymbol{X}_{1}$ y $\boldsymbol{X}_{2}$ la interacción entre la concentración molar y el porcentaje de adición del $\mathrm{Ca}(\mathrm{OH})_{2} \mathrm{y} \xi$ es el error residual experimental (Gutiérrez Pulido \& de la Vara Salazar, 2008) (Caro, 2017). Además, identificada las condiciones para la mejor variable respuesta, se estudia la formación de fases cementantes.

Ecuación 1. Ecuación del modelo de superficie de respuesta.

$$
Y=\beta_{0}+\beta_{1} X_{1}+\beta_{2} X_{2}+\beta_{3} X_{1}^{2}+\beta_{4} X_{2}^{2}+\beta_{5} X_{1} X_{2}+\xi
$$

Los valores del punto central para el hidróxido de calcio se tomaron teniendo en cuenta las investigaciones previas (Bell, 1996) donde se obtuvieron resistencias del orden de $5 \mathrm{MPa}$ con adiciones de $4 \%$ de cal en cuarzo fino, mientras que en suelos arcillosos caoliniticos fue de 1,05 MPa. La concentración molar del activador se toma en cuenta por las investigaciones descritas en los trabajos de Duxson, Criado y otros (Duxson, y otros, 2007) (Criado, 2007) (Fernández-Jiménez, Palomo, \& Criado, 2006), éstos invstigadores establecen que concentraciones del orden de 10 molar, por lo que una variación cercano a este punto puede garantizar la sintesis de el gel NASH. Se toman dos valores encima y dos valores debajo de los puntos medios como lo sugiere el diseño de experimentos (Gutiérrez Pulido \& de la Vara Salazar, 2008). Tabla 3.

\begin{tabular}{|c|c|}
\hline Variables predictoras & Niveles \\
\hline $\begin{array}{l}\text { X1:Concentración Molar } \\
\mathrm{NaOH}\end{array}$ & $\begin{array}{l}\text { 6,58M; 7,0M; 8,0M; 9,0M; } \\
\text { 9,41M }\end{array}$ \\
\hline $\begin{array}{l}\text { X2: Contenido de } \mathrm{Ca}(\mathrm{OH})_{2} \\
\text { en } \%\end{array}$ & $\begin{array}{l}2,1 \% ; 2,5 \% ; 3,5 \% ; 4,5 \% \\
\text { у } 4,9 \%\end{array}$ \\
\hline
\end{tabular}

La matriz experimental (Tabla 4) está constituida por 26 corridas experimentales, en las cuales se incluyen las réplicas y se plantea un diseño central compuesto rotacional para las dos variables predictoras, considerando que los errores presentan una distribución normal N $(0, \sigma 2 I)$. Además, el estimador de máxima verosimilitud de los $\beta$ puede ser usado por medio del método de mínimos cuadrados. Para un mejor tratamiento o combinación de los niveles para las variables predictoras, se han estimado los puntos centrales para cada uno de los factores así: 8 Molar para el $\mathrm{NaOH}$ y $3,5 \%$ en masa del precursor para el $\mathrm{Ca}(\mathrm{OH})_{2}$. Los puntos centrales de cada variable predictora junto con las 10 réplicas son requeridos para validar la variabilidad del proceso en el modelo de superficie de respuesta.

Para esta matriz experimental la prueba de hipótesis nula consiste en que los factores, concentración molar del $\mathrm{NaOH}$ y los porcentajes de adición en masa del $\mathrm{Ca}(\mathrm{OH})_{2}$, no tiene ningún efecto significativo sobre 
la RCS. Para una significancia $\alpha=0,05$, es decir una confianza asociada de $\beta=95 \%$, se puede decir que, si el valor p es menor que $\alpha$, en el análisis ANOVA se rechaza la hipótesis nula.

TABLA 4. MODELO DE MATRIZ PARA DETERMINAR NIVELES DE LOS FACTORES

\begin{tabular}{c|c|c|c}
\hline \multicolumn{2}{|c|}{ Matriz generadora } & \multicolumn{2}{c}{$\begin{array}{c}\text { Valores de los niveles de los } \\
\text { factores }\end{array}$} \\
\hline $\mathrm{Ca}(\mathrm{OH})_{2}$ & $\mathrm{NaOH}$ & $\begin{array}{c}\mathrm{Ca}(\mathrm{OH})_{2} \\
\text { (\% en masa) }\end{array}$ & $\begin{array}{c}\text { NaOH } \\
(\text { Molar) }\end{array}$ \\
\hline 1,000 & $-1,000$ & 4,500 & 7,000 \\
\hline$-1,414$ & 0,000 & 2,086 & 8,000 \\
\hline 0,000 & 0,000 & 3,500 & 8,000 \\
\hline 0,000 & 0,000 & 3,500 & 8,000 \\
\hline 0,000 & $-1,414$ & 3,500 & 6,586 \\
\hline 1,000 & 1,000 & 4,500 & 9,000 \\
\hline 0,000 & 1,414 & 3,500 & 9,414 \\
\hline 0,000 & 0,000 & 3,500 & 8,000 \\
\hline 0,000 & 0,000 & 3,500 & 8,000 \\
\hline 0,000 & 0,000 & 3,500 & 8,000 \\
\hline 0,000 & $-1,414$ & 3,500 & 6,586 \\
\hline 0,000 & 0,000 & 3,500 & 8,000 \\
\hline 0,000 & 0,000 & 3,500 & 8,000 \\
\hline 1,414 & 0,000 & 4,914 & 8,000 \\
\hline 1,414 & 0,000 & 4,914 & 8,000 \\
\hline$-1,000$ & 1,000 & 2,500 & 9,000 \\
\hline$-1,414$ & 0,000 & 2,086 & 8,000 \\
\hline$-1,000$ & $-1,000$ & 2,500 & 7,000 \\
\hline 0,000 & 0,000 & 3,500 & 8,000 \\
\hline$-1,000$ & $-1,000$ & 2,500 & 7,000 \\
\hline$-1,000$ & 1,000 & 2,500 & 9,000 \\
\hline 0,000 & 1,414 & 3,500 & 9,414 \\
\hline 0,000 & 0,000 & 3,500 & 8,000 \\
\hline 1,000 & 1,000 & 4,500 & 9,000 \\
\hline 1,000 & $-1,000$ & 4,500 & 7,000 \\
\hline 0,000 & 0,000 & 3,500 & 8,000 \\
\hline
\end{tabular}

\section{Análisis ANOVA}

Se usa el análisis de varianza (ANOVA) como técnica central para el análisis de datos experimentales que busca "la variación total en las partes con las que contribuye cada fuente de variación en el experimento" (Gutiérrez Pulido \& de la Vara Salazar, 2008) Por lo que se busca identificar si hay efecto por cada tratamiento en la variabilidad total, de tal forma que la variabilidad del error sea más pequeña que la variabilidad debida a los tratamientos. Definida si se acepta o se rechaza la hipótesis nula mediante el uso de ANOVA, se realizan comparaciones de parejas de medias de tratamientos con los valores críticos de cada ecuación, para identificar que tratamientos correspondientes presentan diferencias significativas en la RCS. Se usaron dos métodos el DMS (Diferencia Mínima Significativa) y el de TUKEY. Las ecuaciones por usar son:

Ecuación 2. Ecuaciones de DMS y de TUKEY. Fuente (Gutiérrez Pulido \& de la Vara Salazar, 2008).

$$
\begin{gathered}
D M S=t_{(\propto / 2, N-k)} \sqrt{2 C M_{E} / n} \\
T_{\alpha}=q_{\alpha}(k, N-k) \sqrt{C M_{E} / n}
\end{gathered}
$$

En donde $t_{(\propto / 2, N-k)}$ se lee de las tablas en la distribución T de Student con $N$ - $k$ grados de libertad, que corresponden al error. $C M_{E}$ representa el cuadrado medio del error y se obtiene de la tabla ANOVA y $n$ el número de observaciones. En la ecuación de TUKEY, $k$ es el número de tratamientos, $\alpha$ es el nivel de significancia. También se analizaron los siguientes predictores, el módulo de solución, módulo de sodio y módulo de calcio. Reig y otros (Reig, Soriano, Borrachero, Monzó, \& Payá, 2014), analizaron el comportamiento de las mezclas en diferentes rangos los cuales clasificaron en módulos que se explican en el siguiente numeral.

\section{Módulos de solución, de sodio y de calcio}

El primero de ellos denominado módulo de solución en masa (Ms: Ecuación 3) que relaciona el contenido de $\mathrm{SiO}_{2}$ en la disolución con el contenido de $\mathrm{Na}_{2} \mathrm{O}$ en la disolución. Cuando el módulo de solución es mayor que uno, se presentan tiempo de síntesis (tiempo de fraguado) mayores a 4 horas.

Ecuación 3. Módulo de solución. Tomado de Reig, Soriano, Borrachero, Monzó \& Payá, 2014 


$$
\mathrm{Ms}=\mathrm{SiO}_{2} \text { (disolución) } / \mathrm{Na}_{2} \mathrm{O} \text { (disolución) }
$$

El segundo módulo es llamado módulo de sodio $\left(\mathrm{M}_{\mathrm{Na}}\right.$ Ecuación 4) y relaciona las moles del $\mathrm{Na}^{+}$que son aportadas por el $\mathrm{NaOH}$ según la concentración molary por el silicato de sodio que están presentes en la disolución y que actúan como activadores del $\mathrm{SiO}_{2}$ y el $\mathrm{Al}_{2} \mathrm{O}_{3}$ presentes en el precursor (MK). El incremento de este módulo favorece el aumento de la RCS a edades tempranas.

Ecuación 4 . Módulo de sodio. Tomado de Reig, Soriano, Borrachero, Monzó, \& Payá, 2014.

$$
M_{N a}=N a^{+}(\text {disolución }) /\left(\mathrm{SiO}_{2}+\mathrm{Al}_{2} \mathrm{O}_{3}\right)(\operatorname{del} \mathrm{MK})
$$

El tercer módulo denominado módulo de calcio (MCa), relaciona los moles del Ca2 $2^{+}$y los moles $\mathrm{de} \mathrm{Na}^{+}$ en la disolución activadora. Ver Ecuación 5. Con altas cantidades de $\mathrm{Ca}(\mathrm{OH})_{2}$ y baja concentración de $\mathrm{NaOH}$ se incrementa el valor del módulo $\mathrm{M}_{\mathrm{Ca}}$, favoreciendo un mayor tiempo del CAA en estado fresco.

Ecuación 5. Módulo de calcio. Tomado de Reig, Soriano, Borrachero, Monzó, \& Payá, 2014.

$$
M_{C a}=\mathrm{Ca}_{2}{ }^{+}(\text {Cal hidratada }) / \mathrm{Na}^{+} \text {(disolución) }
$$

La investigación en referencia menciona, que se obtenían rápidas síntesis (fraguados inferiores a 4 horas) cuando se presentaban $\mathrm{Ms}<1$ y $\mathrm{M}_{\mathrm{Na}}>0,2$ y muy poca trabajabilidad de la mezcla fresca cuando $\mathrm{Ms}>2,5 \mathrm{y}$ $\mathrm{M}_{\mathrm{Na}}<0,1$. Por lo cual para un mismo módulo de solución con diferentes módulos de sodio se lograban síntesis de 4 hasta 10 horas. Por otra parte, los valores hallados con los diferentes módulos se aplicaron en el análisis estadístico, se analiza que incidencia tienen cada uno de los módulos con la RCS. De esta forma se puede comparar los resultados hallados de la resistencia a la compresión y la superficie de respuesta y cruzar la información con los módulos que permiten una mayor RCS.

\section{Programa experimental para desarrollar un CAA}

Las mezclas se elaboraron de acuerdo a la Tabla 4 en la amasadora Hobart 1300 se homogenizaron en seco, el MK con la cantidad de hidróxido de calcio especificada en la matriz experimental. Enseguida se vertió lentamente (por 3 minutos) la disolución activadora ( $\mathrm{NaOH}+$
$\mathrm{Na}_{2} \mathrm{SiO}_{3}$ ), se detuvo el proceso para despegar el material adherido a las paredes del recipiente y se continuó hasta completar 5 minutos de mezclado. Para garantizar una pasta homogénea y suelta; antes de compactar, esta se pasó por el tamiz No.4. Luego de homogenizada la mezcla, se compactó en cilindros de $25,4 \mathrm{~mm}$ de radio por $50,8 \mathrm{~mm}$ de alto en moldes de polivinil cloruro (PVC) y se embolsaron para conservar la humedad de mezcla. Posteriormente se llevaron al horno a $40^{\circ} \mathrm{C}$.

Los tiempos de síntesis se dividieron en dos momentos. El primero, fue el tiempo de permanencia del espécimen en bolsa a $40^{\circ} \mathrm{C}$ y comprende el espacio de tiempo desde cuando se terminan de compactar y embolsar las probetas hasta que transcurren 72 horas en un horno BINDER 37059. El segundo momento transcurre cuando, las probetas de pasta endurecida, se extraen del empaque hermético y continúan en el horno a los mismos $40^{\circ} \mathrm{C}$ durante 96 horas más. A cada probeta al término de las 72 horas y las 96 horas se les determinó la humedad y densidad. Finalmente se realizó la prueba de falla a compresión. El equipo utilizado para los ensayos a compresión fue una Humboldt 3300 con una celda de $50 \mathrm{kN}$, la velocidad de ensayo fue de $0,05 \mathrm{~mm} / \mathrm{s}$ para los cilindros, el tiempo no excedió los 15 minutos de ensayo por probeta. La norma usada para el ensayo de la RCS no confinada es la INV E -152.

\section{Resultados y discusión}

\section{del CAA}

\subsection{Resistencia a la compresión simple}

Los resultados de RCS en la matriz de la Tabla 5, indicaron que las resistencias de las pastas del CAA desarrollado oscilaron entre 14 y $32 \mathrm{MPa}$, lo que demuestra que en todos los casos se formaron fases cementantes.

En el análisis de varianza ANOVA para la superficie de respuesta se hace énfasis que los valores $p<0,05$, rechazan la hipótesis nula. Identificando que se rechaza la hipótesis nula, con los factores $\mathrm{NaOH}$ y $\mathrm{Ca}(\mathrm{OH})_{2}$, por lo tanto, la RCS se ve afectada principalmente por la concentración molar del $\mathrm{NaOH}$, que al ser una base fuerte incrementa el pH (>12) generando un mayor rompimiento de enlaces para la formación de cadenas monoméricas de polisiloxanos. El modelo presentó un $\mathrm{R}^{2}=48,36 \%$. 
TABLA 5. MODELO DE MATRIZ PARA DETERMINAR CANTIDADES DE INSUMOS POR PROBETAS DE 2,54 CM DE DIÁMETRO POR 5,08 CM DE ALTO Y EVALUACIÓN DE MÓDULOS DE SOLUCIÓN, SODIO Y CALCIO

\begin{tabular}{|c|c|c|c|c|c|c|c|c|c|}
\hline \multirow{2}{*}{$\begin{array}{c}\text { Orden de } \\
\text { Matriz }\end{array}$} & \multicolumn{3}{|c|}{ Cantidades en gramos } & \multirow{2}{*}{$\begin{array}{c}\begin{array}{c}15 \% \text { en } \\
\text { masa }\end{array} \\
\mathrm{Na}_{2} \mathrm{SiO}_{3}\end{array}$} & \multirow{2}{*}{$\begin{array}{l}\text { Disolución } \\
\text { real por } \\
\text { adicionar (g) }\end{array}$} & \multirow{2}{*}{$\begin{array}{c}\text { Variable } \\
\text { respuesta } \\
\mathrm{MPa}\end{array}$} & \multicolumn{3}{|c|}{ Módulos } \\
\hline & MK & $\mathrm{Ca}(\mathrm{OH})_{2}$ & $\mathrm{NaOH}$ & & & & $M_{s}$ & $M_{\mathrm{Na}}$ & $M_{C a}$ \\
\hline 1 & 181,09 & 8,91 & 62,6 & \multirow{26}{*}{13,5} & \multirow{26}{*}{90} & 15,5 & 0,15 & 0,23 & 0,22 \\
\hline 2 & 185,87 & 4,13 & 71,6 & & & 22,2 & 0,13 & 0,26 & 0,09 \\
\hline 3 & 183,07 & 6,93 & 71,6 & & & 17,1 & 0,13 & 0,26 & 0,15 \\
\hline 4 & 183,07 & 6,93 & 71,6 & & & 16,2 & 0,13 & 0,26 & 0,15 \\
\hline 5 & 183,07 & 6,93 & 58,9 & & & 19,9 & 0,15 & 0,23 & 0,17 \\
\hline 6 & 181,09 & 8,91 & 80,5 & & & 23,4 & 0,12 & 0,28 & 0,17 \\
\hline 7 & 183,07 & 6,93 & 84,2 & & & 21,6 & 0,12 & 0,29 & 0,13 \\
\hline 8 & 183,07 & 6,93 & 71,6 & & & 22 & 0,13 & 0,26 & 0,15 \\
\hline 9 & 183,07 & 6,93 & 71,6 & & & 22,7 & 0,13 & 0,26 & 0,15 \\
\hline 10 & 183,07 & 6,93 & 71,6 & & & 19,5 & 0,13 & 0,26 & 0,15 \\
\hline 11 & 183,07 & 6,93 & 58,9 & & & 19,8 & 0,15 & 0,23 & 0,17 \\
\hline 12 & 183,07 & 6,93 & 71,6 & & & 22,3 & 0,13 & 0,26 & 0,15 \\
\hline 13 & 183,07 & 6,93 & 71,6 & & & 20,8 & 0,13 & 0,26 & 0,15 \\
\hline 14 & 180,27 & 9,73 & 71,6 & & & 20 & 0,13 & 0,26 & 0,21 \\
\hline 15 & 180,27 & 9,73 & 71,6 & & & 19 & 0,13 & 0,26 & 0,21 \\
\hline 16 & 185,05 & 4,95 & 80,5 & & & 21,8 & 0,12 & 0,28 & 0,1 \\
\hline 17 & 185,87 & 4,13 & 71,6 & & & 14,8 & 0,13 & 0,26 & 0,09 \\
\hline 18 & 185,05 & 4,95 & 62,6 & & & 15,6 & 0,15 & 0,23 & 0,12 \\
\hline 19 & 183,07 & 6,93 & 71,6 & & & 23 & 0,13 & 0,26 & 0,15 \\
\hline 20 & 185,05 & 4,95 & 62,6 & & & 19 & 0,15 & 0,23 & 0,12 \\
\hline 21 & 185,05 & 4,95 & 80,5 & & & 25 & 0,12 & 0,28 & 0,1 \\
\hline 22 & 183,07 & 6,93 & 84,2 & & & 32,8 & 0,12 & 0,29 & 0,13 \\
\hline 23 & 183,07 & 6,93 & 71,6 & & & 22,6 & 0,13 & 0,26 & 0,15 \\
\hline 24 & 181,09 & 8,91 & 80,5 & & & 31,5 & 0,12 & 0,28 & 0,17 \\
\hline 25 & 181,09 & 8,91 & 62,6 & & & 21,8 & 0,15 & 0,24 & 0,21 \\
\hline 26 & 183,07 & 6,93 & 71,6 & & & 24,9 & 0,13 & 0,26 & 0,15 \\
\hline
\end{tabular}

\subsection{Resultados de los Módulos de solu-} ción, de sodio y de calcio en CAA

Los resultados de los módulos de solución, sodio y calcio se muestran en la Tabla 5, e indicaron que las resistencias de las pastas del CAA desarrollado oscilaron entre 14 y $32 \mathrm{MPa}$, lo que demuestra que en todos los casos se formaron fases cementantes.

Todos los valores de $\mathrm{M}_{\mathrm{S}}$ se hallan por debajo del valor de 1,0 y todos los valores de $\mathrm{M}_{\mathrm{Na}}$ se encuentren por encima de 0,2, garantizando que la mezcla permanecerá en estado fresco hasta por 4 horas. (Reig, Soriano, Borrachero, Monzó, \& Payá, 2014). Al análisis ANOVA con el uso de estos factores ratifican que la molaridad del $\mathrm{NaOH}$ y la adición del $\mathrm{Ca}(\mathrm{OH}) 2$ afectan la variable respuesta.

\subsection{Análisis DRX del CAA}

Las probetas que mejor desempeño mecánico presentaron en la campaña experimental del modelo de superficie de respuesta del MK activado definen las 
siguientes condiciones: 9,4M de $\mathrm{NaOH}$ y 4,5\% de adición de $\mathrm{Ca}(\mathrm{OH})_{2}$. Los DRX de las pastas al CAA presentan contenidos de cuarzo, material que no reacciona fácilmente con la disolución activadora por la naturaleza cristalina. El código del cuarzo es el PDF 01-083-0539 y los picos representativos se hallaron en los ángulos $20,83^{\circ}, 26,59^{\circ}$, $36,49^{\circ}, 39,39^{\circ}, 40,22^{\circ}, 42,38^{\circ}, 45,72^{\circ}$ y $50,05^{\circ}$ del MK activado. Además, la reacción del MK con el $\mathrm{NaOH}$ y el silicato de sodio, hace que se presente un pico ancho en $30^{\circ}$ de 2 theta, característico de los geopolímeros sin importar el contenido de $\mathrm{Al}$ o Si (Provis, Lukey, \& Deventer, 2005) (De Silva, Sagoe-Crenstil, \& Sirivivathanon, 2007) (Hoyos Montilla, Arias Jaramillo, \& Tobón, 2018). Los resultados de DRX son acordes a (Li, Sun, \& Li, 2010), quien afirma que al mezclarse el $\mathrm{NaOH}$ y el $\mathrm{Na}_{2} \mathrm{SiO}_{3}$, el material formado es un gel amorfo NASH, con una composición química similar a las zeolitas, pero sin una estructura cristalina extensa.
Las fases cristalinas minoritarias producto de la síntesis a 72 horas, se presentan en la Figura 2, donde se evidencian zeolitas tipo A $\left(\mathrm{Na}_{2} \mathrm{Al}_{2} \mathrm{Si}_{11,85} \mathrm{O}_{7,7} 5,1\left(\mathrm{H}_{2} \mathrm{O}\right)\right.$ código de referencia PDF 00-038-0241 en los ángulos $7,19^{\circ}, 10,15^{\circ}, 12,44^{\circ}, 16,07^{\circ}, 21,62^{\circ}, 23,94^{\circ}, 26,06^{\circ}, 27,06^{\circ}$, $29,89^{\circ}, 32,49^{\circ}, 34,12^{\circ}, 42,11^{\circ}, 52,46^{\circ}$. Estas zeolitas son tectosilicatos, lo que garantiza una unión continua y tridimensional de los tetraedros de sílice, siendo la característica principal de la formación de fases cementantes producto de los cementos alcalinos. Se podría afirmar que la presencia de zeolitas está fuertemente ligada a la formación de estructuras tridimensionales en el proceso de síntesis desarrollando una estructura policristalina que incrementa las propiedades mecánicas (Komnitsas Kostas \& Zaharaki Dimitra, 2007). Según Xu en el 2001, (Xu H \& Van Deventer, 2001).
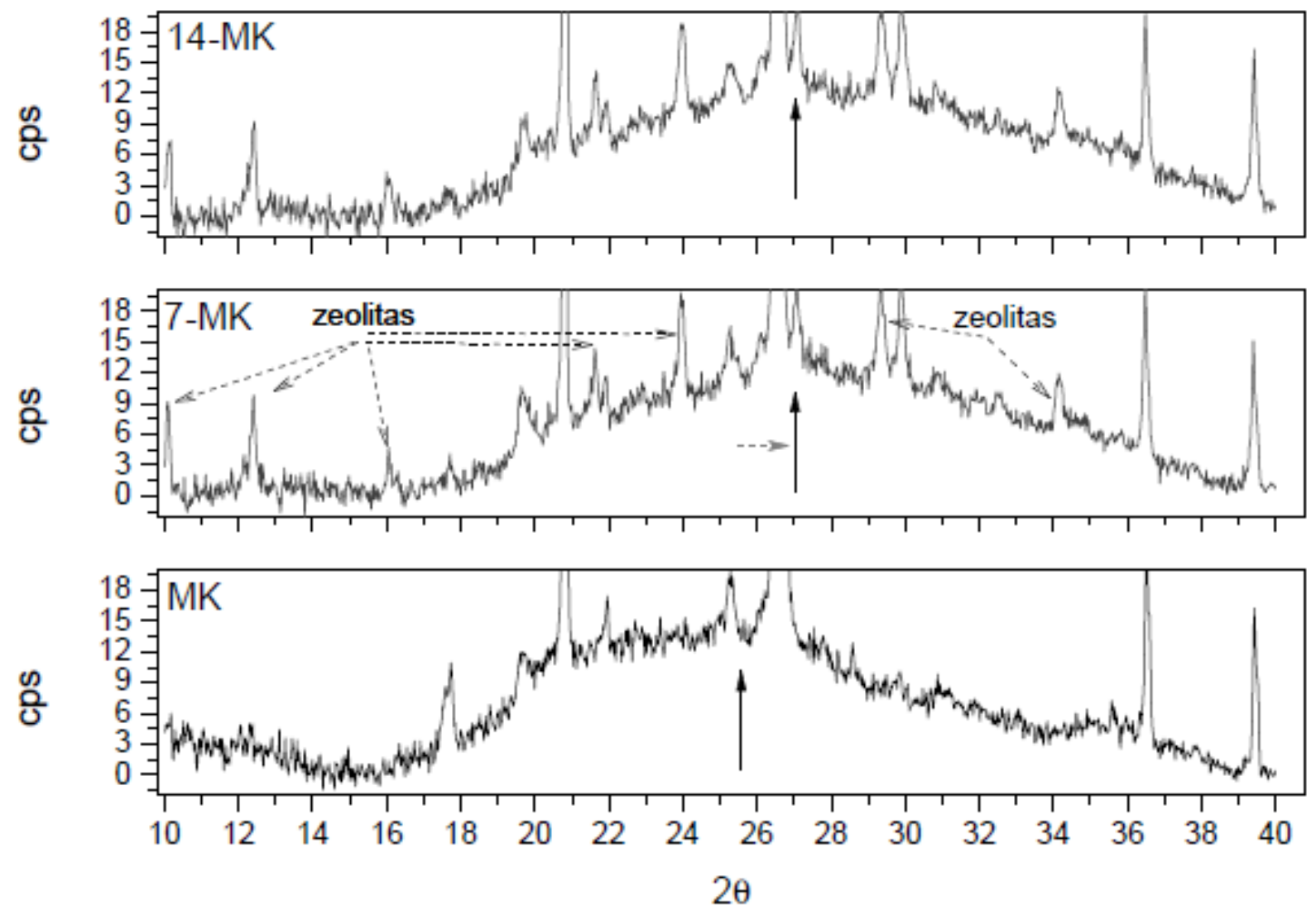


\section{Análisis de la superficie de respuesta}

El análisis de la superficie de respuesta se realizó teniendo en cuenta que los factores son: concentración molar del $\mathrm{NaOH}$ y porcentaje de adición en masa del $\mathrm{Ca}(\mathrm{OH})_{2}$, sobre la RCS no confinada como variable respuesta (Figura 3). Una descripción de los efectos principales sobre la variable respuesta y las interacciones entre los factores se menciona a continuación.

\section{Figura 3. Superficie de respuesta de la RCS en probetas} de CAA

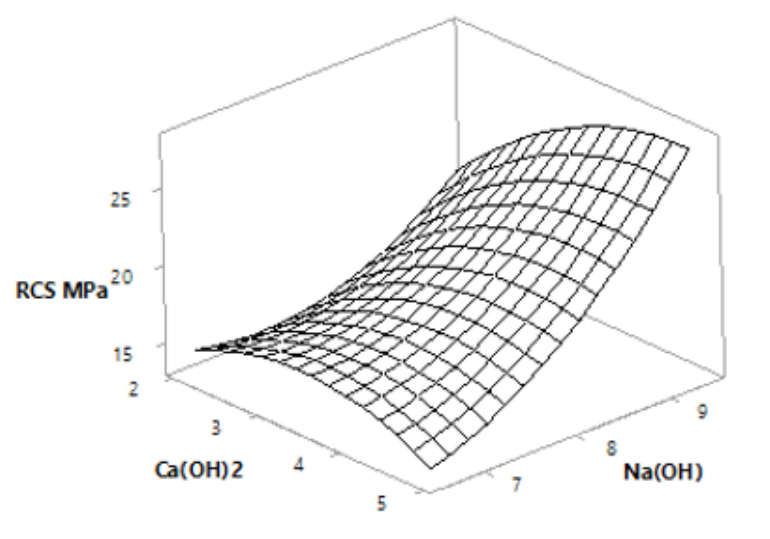

\section{Figura 4. Relación del $\mathrm{NaOH}$ y el $\mathrm{Ca}(\mathrm{OH})_{2}$ en la $\mathrm{RCS}$ con pro-} betas de CAA

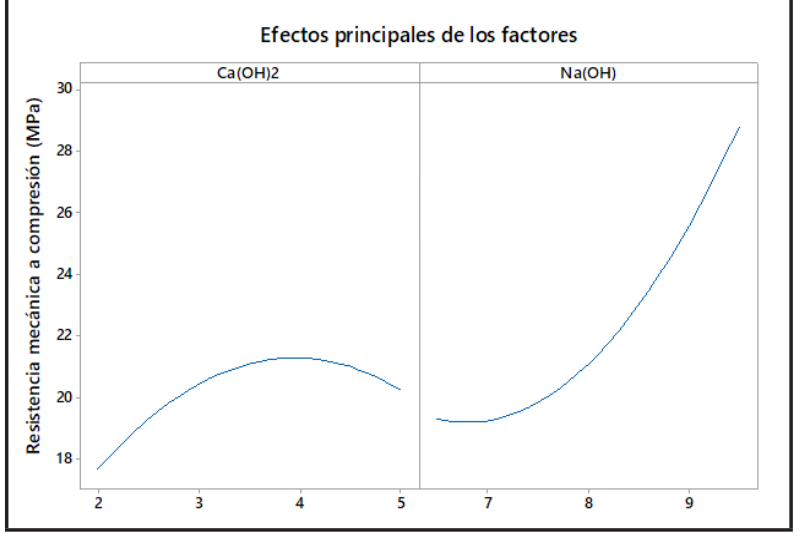

Efectos principales de los factores. De la Figura 4, se observa que al evaluar el efecto principal del factor $\mathrm{NaOH}$ el incremento en la concentración molar presenta una relación directa con el aumento de la RCS, presentándose principalmente para valores en concentraciones superiores a $8 \mathrm{M}$, valores inferiores presentan un efecto más reducido, esto puede estar asociado a la baja disponibilidad de grupos $(\mathrm{OH})$ para que el sistema genere rompimiento de los enlaces y se dé el posterior desarrollo del gel NASH. En cuanto al factor $\mathrm{Ca}(\mathrm{OH})_{2}$, se presenta un máximo en la RCS alrededor del 4\% de adición en masa indicando un efecto de curvatura en la disminución de la RCS a mayores valores.

Interacción de los factores: En la Figura 5, el incremento de la RCS se presentó con pequeñas adiciones de $\mathrm{Ca}(\mathrm{OH})_{2}$. Es así como el punto máximo del $\mathrm{Ca}(\mathrm{OH})_{2}$ se desplaza hacia la derecha, cuando se aumenta la concentración molar del $\mathrm{NaOH}$, indicando las proporciones que ambos activadores deberán tener para conformar los productos de reacción con las más altas RCS.

Figura 5. Iteración del $\mathrm{NaOH}$ y $\mathrm{Ca}(\mathrm{OH})_{2}$ frente a la variable respuesta RCS

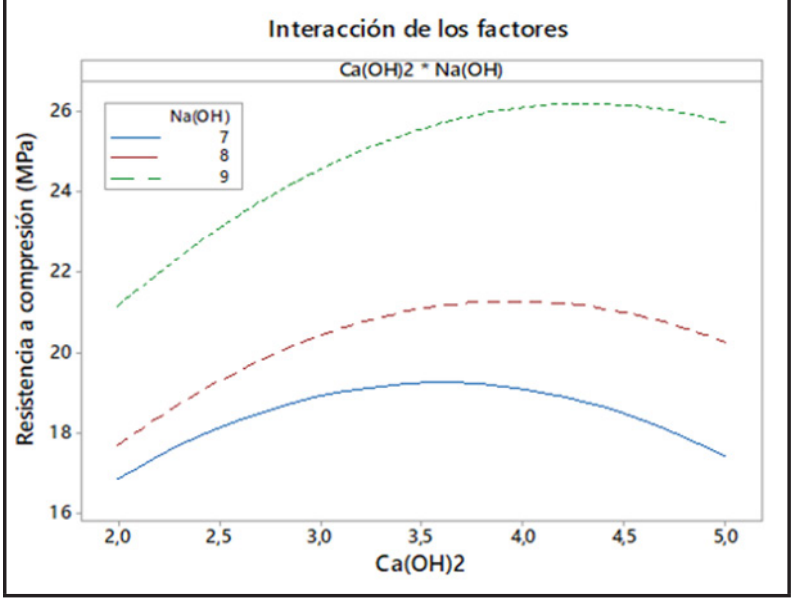

8.1. Diferencias significativas en la RCS, mediante el DMS (Diferencia Mínima Significativa) y el de TUKEY

Con el objetivo de identificar los grupos más confiables en el diseño experimental, se usó el método de MDS (Mínima Diferencia Significativa, por sus siglas en ingles) y el de TUKEY, hallando los valores de la Tabla 6. Existe, una marcada diferencia entre las medias de los siguientes tratamientos: $6,6 \mathrm{M} \neq$ $9 M, 6,6 M \neq 9,4 M, 7 M \neq 9 M, 7 M \neq 9,4 M, 8 M \neq 9,4 M$ y se concluye que los grupos más confiables son de 9 y 9,4 molar del $\mathrm{NaOH}$ según el DMS. 
TABLA 6. RESULTADOS PARA COMPARACIÓN DE PAREJAS DE TRATAMIENTO

\begin{tabular}{c|c|c|c|c|c|}
\hline Notación & Diferencia de medias & \multicolumn{2}{|c|}{ DMS } & \multicolumn{2}{c}{ TUKEY } \\
\hline $6,6 \mathrm{M}-7 \mathrm{M}$ & 2,75 & $<6,99$ & No significativa & $<9,98$ & No significativa \\
\hline $6,6 \mathrm{M}-8 \mathrm{M}$ & 4,42 & $<6,99$ & No significativa & $<9,98$ & No significativa \\
\hline $6,6 \mathrm{M}-9 \mathrm{M}$ & 11,02 & $>6,99$ & Significativa & $>9,98$ & Significativa \\
\hline $6,6 \mathrm{M}-9,4 \mathrm{M}$ & 12,15 & $>6,99$ & Significativa & $>9,98$ & Significativa \\
\hline $7 \mathrm{M}-8 \mathrm{M}$ & 1,67 & $<6,99$ & No significativa & $<9,98$ & No significativa \\
\hline $7 \mathrm{M}-9 \mathrm{M}$ & 8,27 & $>6,99$ & Significativa & $<9,98$ & No significativa \\
\hline $7 \mathrm{M}-9,4 \mathrm{M}$ & 9,40 & $>6,99$ & Significativa & $<9,98$ & No significativa \\
\hline $8 \mathrm{M}-9 \mathrm{M}$ & 6,60 & $<6,99$ & No significativa & $<9,98$ & No significativa \\
\hline $8 \mathrm{M}-9,4 \mathrm{M}$ & 7,73 & $>6,99$ & Significativa & $<9,98$ & No significativa \\
\hline $8 \mathrm{M}-9,4 \mathrm{M}$ & 1,13 & $<6,99$ & No significativa & $<9,98$ & No significativa \\
\hline
\end{tabular}

\section{Conclusiones}

En la región explorada se obtuvieron resistencias a compresión de la pasta entre 15 y 34,6 MPa, lo que demostró que la síntesis del cemento alcalino es factible para el precursor de metacaolín de industria colombiana. Las mayores resistencias se lograron bajo la condición de 9 molar de $\mathrm{NaOH}, 4,5 \%$ de $\mathrm{Ca}(\mathrm{OH})_{2}, 15 \%$ de $\mathrm{Na}_{2} \mathrm{SiO}_{3}$ y los $68,06 \%$ en masa del MK. La técnica de DRX, a los 7 días de síntesis, permitió validar la formación de fases cementantes en la pasta del CAA mediante el desplazamiento del halo amorfo entre 18 y $30^{\circ}$ en 2 theta. Aunque la presencia de zeolitas no favorece las propiedades mecánicas de los cementos alcalinos, éstas dan cuenta de la formación de tectosilicatos siendo característico de fases cementantes que se extiende a la fase amorfa.

El diseño de superficie de respuesta permitió identificar la interacción de los factores $\mathrm{NaOH}$ y $\mathrm{Ca}(\mathrm{OH})_{2}$. Donde la respuesta mecánica de la pasta evaluada evidenció un efecto en la curvatura para el aumento en concentración de $\mathrm{Ca}(\mathrm{OH})_{2}$, esto puede ser atribuido probablemente a la reducción de la solubilidad del Ca y el incremento de la concentración de Si, Mientras que la concentración de $\mathrm{Na}(\mathrm{OH})$ generó un efecto muy marcado en el aumento de las propiedades mecánicas. Siendo el $\mathrm{Na}(\mathrm{OH})$ el factor principal en la formación de fases cementantes, esto por ser una base fuerte que permite un mejor rompimiento de los enlaces $\mathrm{Si}-\mathrm{O}, \mathrm{Al}-\mathrm{O}$. Mediante el uso del análisis ANOVA para el modelo propuesto, se identificó que la concentración molar es la variable pre- dictiva que más significancia presenta con la resistencia a la compresión simple (RCS) para desarrollar un CAA, porque rechaza la hipótesis nula. Este comportamiento se reafirmó con el análisis ANOVA, donde la variable $\mathrm{M}_{\mathrm{Na}}$ presenta la mayor correlación con RCS.

En tanto que la superficie de respuesta $\left(R^{2}=0,48\right)$ solo acepta la variable concentración molar $(\mathrm{NaOH})$ para rechazar la hipótesis nula. Al incluir la densidad y la humedad a un nuevo modelo, quedan 4 variables (densidad, humedad, $\mathrm{NaOH}$ y la cal) que son analizadas por la regresión lineal, indicando que todas las variables predictivas son significativas $\left(R^{2}=0,63\right)$. Además, la variable predictiva densidad presenta la mayor correlación con la variable respuesta RCS.

Con base en el método de la Mínima Diferencia Significativa (MDS), se identificó que existe una marcada diferencia entre las medias de los grupos que conforman las concentraciones molares del $\mathrm{NaOH}$ y la RCS, indicando que las máximas RCS se presentan cuando existe una concentración del $\mathrm{NaOH}$ mayor o igual a 9 molar.

\section{Agradecimientos}

Los autores de este trabajo agradecen a los laboratorios de la Universidad de Medellín.

\section{Referencias}

Alonso S., \& Palomo A. (2000). Alkaline activation of mettakaolin and calcium hydroxide mixtures: influence of 
temperature, activator concentration and solids ratio. MATERIALS LETTERS.

Bell, F. (1996). Lime stabilization of clay minerals and soils. Engineering Geology, 223-237.

Bernal S.A., Mejía R., Provis J.L., \& Rose V. (2010). Effect of silicate modulus and metakaolin incorporation on the carbonation of alkali silicate-activated slags. Cement and Concrete Research.

Caro, F. (3 de 05 de 2017). Estadistica. (C. A. Villamil, Entrevistador)

Criado, M. S. (2007). Nuevos materiales cementantes basados en la activación alcalina de cenizas volantes. Caracterización de geles $N-A-S-H$ en función del contenido de Sílice soluble. Efecto del Na2SO4. Madrid, España: UNIVERSIDAD AUTONOMA DE MADRID.

Davidovits, J. (1991). GEOPOLYMERS: INORGANIC POLYMERIC NEW MATERIALS. Geopolymer Instute 1997, 1633-1656.

De Silva, P., Sagoe-Crenstil, K., \& Sirivivathanon, V. (2007). Kinetics of geopolymerization: Role of $\mathrm{Al} 2 \mathrm{O} 3$ and $\mathrm{SiO} 2$. Cement and Concrete Research, 512-518.

Diamond, S. (1982). ON THE GLASS PRESENT IN LOWCALCIUM. School of Civil Engineering-Purdue University, West Lafayette, Indiana, 459-464.

Duxson, P., Fernández-Jiménez, A., Provis, J., Lukey, G., Palomo, A., \& van Deventer, J. (2007). Geopolymer technology: the current state of the art. Journal of material science, 2917-2933.

Fernández-Jiménez, A., Palomo, A., \& Criado, M. (2006). Alkali activated fly ash blinder. A comparative study between sodium and potassium activators. Materiales de Construcción, 51-65.

Gutiérrez Pulido, H., \& de la Vara Salazar, R. (2008). Análisis $y$ diseño de experimentos. México, D.F.: McGraw-Hill Interamericana.

Hoyos Montilla, A., Arias Jaramillo, Y., \& Tobón, J. (2018). Evaluation of cements obtained by alkali-activated coal ash with $\mathrm{NaOH}$ cured at low temperatures. $M a-$ teriales de construcción,, V.68.

INSUMOS INDUSTRIALES CORONA. (12 de OCTUBRE de 2013). ESPECIFICACIONES DE PRODUCTO. METACAOLIN DE ALTA REACTIVIDAD PUZOLÁNICA. MEDELLIN, ANTIOQUIA, COLOMBIA: .

Khale, D., \& Chaudhary, R. (2007). Mechanism of geopolymerization and factors influencing its development: a review. Journal of Materials Science, 729-746.

Komnitsas Kostas, \& Zaharaki Dimitra . (2007). Geopolymerisation: A review and prospects for the minerals industry. Science Direct, 1261-1277.
Lecomte, I., Liégeois, M., Rulmont, A., \& Cloots, R. (2003). Synthesis and characterization of new inorganic polymeric composites based on kaolin or white clay and on ground-granulated blast furnace slag. Journal of Materials Research , 2571-2579.

Li, C., Sun, H., \& Li, L. (2010). A review: The comparison between alkali-activated slag $(\mathrm{Si}+\mathrm{Ca})$ and metakaolin (Si+Al) cements. Cement and Concrete Research, 1341.

Liew Yun-Ming, Heah, C.-Y., Mohd, M., \& Kamarudin, H. (2016). Structure and properties of clay-based geopolymer cements: A. Progress in Materials Science, 595-629.

Nagaraj, H., Rajesh, A., \& Sravan, M. (2016). Influence of soil gradation, proportion and combination of admixtures on the properties and durability of CSEBs. Construction and Building materials, 135-144.

Nagaraj, H., Sravan, M., Arun, T., \& Jadadish, K. (2014). Role of lime with cement in long-term strength of compressed stabilized earth blocks. international journal of sustainable built environment, 54-61.

Palomo, A., Krivenko, P., Garcia-Lodeiro, I., Kavalerova, E., Maltseva, O., \& Fernández-Jiménez A. (2014). A revie on alkaline activation: new analytical perspectives. MATERIALES DE CONSTRUCCIÓN.

Palomo, A., Macias, A., Blanco, M., \& Puertas, F. (1992). Physical chemical and mechanical characterization of geopolymers. 9th International congress on the chemestry of cement, (págs. 505-511). New Delhi.

Penman, A. (1989). Dams, In Civil engineer's Reference Book. Butterworth - Heinemann: LS Blake.

Provis, J., Lukey, G., \& Deventer, J. (2005). Do geopolymers actually contain nanocrystalline zeolites? Chemical Mater, 3075-3085.

Provis, J., Palomo, A., \& Shi, C. (2015). Advances in understanding alkali-activated materials. Cement and Concrete Research, 110-125.

Reig, L., Soriano, L., Borrachero, M., Monzó, J., \& Payá, J. (2014). Influence of the activator concentration and calcium hidroxide addition on the properties of alkaliactived porcelain waste. Constrution and building materials, 214-222.

Rowshanzamir, M., \& Askari, A. (2010). An investigation on the strength anisotropy of compacted clays. Applied Clay Science, 520-524.

Tripura, D., \& Konjengbam, D. (2015). Axial load-capacity of rectangular cement stabilized rammed earth. Engineering Structures, 402-412. 
Venkatarama Reddy, B., \& Latha, M. (2014). Retrieving clay minerals from stabilised soil compacts. Applied Clay Science, 362-368.

Villa, C., Pecina, E., Torres, R., \& Gómez, L. (2010). Geopolymer synthesis using alkaline activation of natural zeolite. Construction and Building Materials, 2084-2090.

Xu H, \& Van Deventer, J. (2001). Geopolymerisation of aluminosilicate minerals. Melboure, Australia: Department of Chemical Engineering, University of Melbourne.

Yip C.K., Lukey G.C., \& van Deventer J.S.J. (2004). The coexistence of geopolymeric gel and calcium silicate hydrate at the early stage of alkaline activation. Cement and Concrete Research.

PARA CITAR ESTE ARTÍCULO TO REFERENCE THIS ARTICLE / PARA CITAR ESTE ARTIGO /

Díaz-Villamil, C.A.; Alzate-Ramirez, A.L. Arias-Jaramillo, Y.P. (2020). Evaluación mecánica y mineralógica de un cemento de metacaolín utilizando el método de superficie de respuesta. Revista EIA, 17(33) enero-junio, Reia33009 pág. 1-12. Disponible en: https://doi.org/10.24050/reia. v17i33.1336 